\title{
Las narrativas visuales y audiovisuales de ficción y la educación artística identitaria
}

\author{
Ricard Ramon \\ Universitat de València (España)
}

\begin{abstract}
El artículo plantea un análisis sobre la manera en que las narrativas visuales y audiovisuales de ficción ofrecen una serie de oportunidades educativas para poder trabajar diferentes problemáticas de la identidad. Explora las posibilidades que la ficción artística tiene para generar múltiples construcciones posibles de identidades, como la diversidad sexual y de género entre muchas otras, favoreciendo la comprensión empática de los otros y permitiendo una mejor relación entre seres humanos. Entendemos las narrativas de ficción como una expresión artística constructora de verdades simbólicas necesarias para la comprensión profunda del mundo y de las personas. Son por tanto un medio de aprendizaje artístico. Se desarrolla un análisis teórico sobre el concepto de ficción y se reivindica como instrumento educativo y se analizan ejemplos de artistas y de experiencias de exploración identitaria y de autoconocimiento a través de este tipo de prácticas. Como conclusión, el artículo establece que las narrativas de ficción contribuyen de forma creativa y eficaz a la comprensión de formas de vida e identidades diferentes, permiten experimentar sobre la propia identidad $\mathrm{y}$, por tanto, son un instrumento de aprendizaje sobre uno mismo y los demás.
\end{abstract}

Palabras clave: Narrativas, ficción, educación artística, identidades, diversidad.

The visual and audiovisual fiction narratives and art education of identity. The article presents an analysis of the manner in which the visual narratives and audiovisual fiction offer a range of educational opportunities in order to be able to work on different problems of identity. Explores the possibilities that the artistic fiction has to generate multiple possible constructions of identities, for example, sexual and gender diversity among many other, favouring the empathic understanding of others and allowing a better relationship between human beings. We understand the narratives of fiction as an artistic expression builder of symbolic truths necessary for the understanding of the world and of people. They are therefore a means of artistic learning. Develops a theoretical analysis of the concept of fiction and is claimed as an educational tool and discusses examples of artists and experiences of identity and self-exploration through this type of practices. In conclusion, the article provides that the narratives of fiction contribute in a creative and effective way to the understanding of life forms and different identities, allow you to experiment on their own identity and, therefore, are a tool for learning about oneself and others.

Keywords: Narratives, fiction, art education, identities, diversity.

Correspondencia: Ricard Ramon. Universitat de València. Avda. Blasco Ibáñez, no 13. C.P.: 46010. Valencia (España). E-mail: $\underline{\text { ricard.ramon@uv.es }}$ 
A través de este texto, se aborda un relato que, en primer lugar, analiza la forma en la que las narrativas de ficción contribuyen a mejorar y a crear climas de sensibilización y prácticas empáticas en cuestiones vinculadas a la identidad, la diversidad de género y la diversidad sexual. Y, por otro lado, explora las posibilidades que las prácticas artísticas, creativas e imaginativas y las pedagogías visuales ofrecen como recurso educativo en la construcción libre de las identidades.

Las identidades, y especialmente las identidades individuales que tienen que ver con la afirmación del individuo en cuestiones relacionadas con el género o la opción sexual con la que cada persona siente su realización de forma plena, son aspectos obviamente muy vinculados al ámbito educativo. En este sentido, podemos destacar las aportaciones que el profesor Huerta ha realizado al respecto a través de numerosos artículos y especialmente de los libros Transeducar (Huerta, 2016) y Educación artística $y$ diversidad sexual (Huerta y Alonso-Sanz, 2015), -en este caso en un libro colectivo coordinado junto a la profesora Amparo Alonso,- que suponen el primer intento serio y riguroso de conjugar las posibilidades que las prácticas, experiencias e investigaciones artísticas poseen como vía para el desarrollo de cambios sociales e individuales con repercusión positiva en la construcción, afirmación y percepción de diferentes modelos identitarios del ser humano.

Como instrumento predilecto para trabajar todas estas cuestiones, partimos de las narrativas, un concepto que engloba un amplio campo de prácticas y significados, muchos de ellos vinculados a la creación y generación de nuevos significados, y tal y como afirma la profesora Agra (2005):

En suma, "la narrativa es una capacidad humana fundamental y ahí radica su importancia en el campo de la educación. En un sentido amplio, podemos afirmar que las personas, en su relación con los demás, y consigo mismos, no hacen otra cosa que construir - contar, soñar, imaginar, dibujar, recordar, creer, dudar, planificar, vivir...- sus propias narrativas. Narrar es entonces un modo básico de recrear la realidad, es decir, de conocer. El enfoque narrativo se centra en el relato -narración- como un género específico de discurso; aquel que expresa una experiencia humana relevante". (p. 137)

Las narrativas de ficción constituyen un campo de investigación dentro de las Ciencias Sociales, con un cierto recorrido y una base argumental, metodológica y práctica bastante sólida en la actualidad, aunque todavía con ciertas carencias derivadas de determinados complejos, que se resisten a desaparecer frente a la visión científica y positivista del conocimiento. La creación de conocimiento, es decir, el aprendizaje es algo muy complejo multidimensional, y difícilmente, por no decir de forma imposible, se puede reducir a los parámetros tan limitados y estrictos que el positivismo plantea.

Leavy (2018) escribe y articula, en un texto muy reciente, toda una estructura de cómo se puede trabajar la investigación en Ciencias Sociales, desde las narrativas de ficción, destacando su conexión con elementos auto-etnográficos, trabajando cuestiones 
relacionadas con la identidad y argumentando sobre la importancia educativa que las narrativas de ficción tienen en la creación de la empatía y la comprensión de otras realidades e identidades: Cultivating empathy can be an integral part of research practice across the disciplines. Whether we are conducting research about surviving trauma, bullying, or violence; eating disorders; living with or caring for someone with a terminal illness; imprisonment; experiences of racism, sexism, or homophobia; immigration or many other topics, it is customary to want to take what we learn and share it with others in a way that illuminates and makes people more sympathetic to the needs and experiences of others. When we learn about people and their situations, there is the possibility of enlarging our understanding of the world. We may become more understanding, tolerant, and open to the needs and perspectives of those with whom we share differences. Fiction can allow us, metaphorically, to walk in the shoes of another and is therefore uniquely well suited to the promotion of empathy. (Leavy, 2018, p. 194)

Este es uno de los argumentos fundamentales a la hora de trabajar con este modelo, centrado en el uso pedagógico y de investigación que la ficción narrativa nos ofrece, en todas sus variantes. Si bien es cierto que tradicionalmente, las narrativas de ficción se han visto casi siempre vinculadas al ámbito de la literatura, en nuestro caso, como especialistas en el ámbito de lo visual, entendemos que la ficción es un campo especialmente prolífico en el ámbito de los lenguajes visuales y audiovisuales. Todas estas prácticas artísticas poseen un correlato natural en la creación, producción e investigación educativa mediante imágenes estáticas o en movimiento.

La ficción, se define habitualmente en contraposición a aquello que es real, y se entiende de forma habitual como todo lo que no responde a hechos acontecidos, lugares que existen físicamente en un espacio geográfico concreto, animales plantas y objetos identificables y reconocibles, sucesos factiblemente reconocidos por el pensamiento dominante, o el pensamiento científico dominante, incluso pensamientos y estructuras de pensamiento derivadas del paradigma predominante, entre otras cosas. Todos ellos son aspectos que se consideran ficción, en un sentido peyorativo y dando por sentado que todo aquello que responde al mundo físico, material y geográfico, a los hechos acontecidos, incluso a los hechos que se espera sean acontecidos en función de una serie de parámetros del imaginario social sobre lo que se espera que ocurra en determinadas circunstancias y situaciones o rigiéndose bajo leyes físicas o científicas, es la no ficción.

En un sentido estricto, la ficción es definida por la Real Academia de la Lengua Española de la siguiente manera: "1. f. Acción y efecto de fingir. 2. f. Invención, cosa fingida. 3. f. Clase de obras literarias o cinematográficas, generalmente narrativas, que tratan de sucesos y personajes imaginarios." Es decir, que históricamente la ficción se asocia a la mentira, a la falta de verdad o de autenticidad. Un escenario muy negativo del que se parte a la hora de reivindicar la ficción como un instrumento de creación de 
conocimiento, ya que se asociará a un tipo de conocimiento supuestamente falso o falseado.

En ningún caso tratamos de demostrar que no existen la verdad o la falsedad desde un relativismo radical, en absoluto, más bien partimos de que muchas de las cosas que entendemos como ficción parten de una verdad mucho más profunda, especialmente la ficción narrativa y creativa con contenido estético, y las enmarcamos en la posición filosófica sobre la cognición que plantea el filósofo Goodman (1995, p. 33), cuando hace referencia a como, especialmente en aquello referido a los modos de conocer y aprehender el mundo "de que no existe ningún modo correcto de describir, pintar o percibir 'el mundo', sino que existen, más bien, muchos modos igualmente correctos, aunque entren en conflicto -y por consiguiente, en efecto, muchos mundos reales." Y continua, sin negar la existencia de versiones falsas, insistiendo en que: "Las versiones que no se aplican al mismo mundo no entran en conflicto; la segregación evita la contradicción. Una versión verdadera es verdadera en algunos mundos, una versión falsa en ninguno. De este modo, los múltiples mundos de las versiones verdaderas que entran en conflicto son los mundos reales y no los mundos meramente posibles, ni los no mundos de las versiones falsas. Por tanto, si hay algún mundo real, entonces hay muchos. Porque hay versiones verdaderas en conflicto y éstas no pueden ser verdaderas en el mismo mundo. Aunque el concepto de una multiplicidad de mundos reales es extraña e inconcebible, parece que nos vemos obligados, no obstante, a mantenerlo, frente a la alternativa inaceptable de un mundo en el que lo contradictorio y, por tanto, todas las versiones, fueran verdaderas." (Goodman, 1995, p. 60)

Pero además de ellos y sin lugar a duda, la ficción, cuando es articulada a través de narrativas, obliga en primer lugar a la creación y activación de pensamiento. La narrativa supone un pensamiento estructurado y secuenciado, obliga a dotar de sentido, de significados y simbolismos la construcción ficticia, que no deja de ser una verdad sobre nosotros mismos, una parte de nosotros mismos o una parte del mundo percibida por nosotros, que estamos elaborando.

En segundo lugar, lo que podríamos llamar como una narrativa de lo verdadero que no deja de ser otra cosa que una mera descripción, del mundo o de los acontecimientos. Incluso esta descripción supuestamente objetiva necesita de recursos narrativos para poder dotar de un sentido mínimo, un sentido que no deja de ser simbólico y con un alto grado de abstracción. Es decir, ficción narrativa frente a descripción, aquello que llamamos verdadero no deja de ser una interpretación simbólica-narrativa del mundo, una abstracción simplificada de la realidad, que nos permite comprenderla y reconstruirla.

Si estas narrativas se construyen mediante la aportación de lenguajes visuales, como los desarrollados por Mesias-Lema en el ámbito de la fotografía como medio narrativo en educación (2008) o los lenguajes audiovisuales, como el trabajo de García (2012) con el videoarte en contextos educativos, añadimos una significación simbólica 
aún mayor y aumentamos las posibilidades de narrar, de construir múltiples visiones del mundo, mucho más sutiles, abiertas, multidimensionales, poéticas y especialmente, mucho más sensibles y estéticas. Con ello, es incluso más sencillo construir procesos de relación empática y de sensibilización y comprensión de los mundos del otro y de uno mismo, de una forma más contundente y compleja, y también más significativa.

Muchas veces la crudeza de las palabras no nos permite construir, mediante un lenguaje textual articulado, aquello que deseamos narrar, o nuestra inteligencia visualespacial es superior a nuestra inteligencia verbal-lingüística (Gardner, 2014), y la estética de las imágenes nos ofrece entonces el mejor camino para construir relatos identitarios a partir de una ficción que siempre se construye en base a una parte real del mundo y de nosotros mismos.

No se trata de que a través de lo visual se narre lo que les sucede a los seres humanos, sino de que en su forma narrativa capte una forma que ya estaba presente en las vidas relatadas. Una narrativa, trata no sólo de hechos, ideas o teorías, o hasta de sueños, temores y esperanzas, sino de hechos, teorías y sueños desde la perspectiva de la vida de alguien y dentro del contexto de las emociones de alguien. Es conveniente recordar que todo el conocimiento que tenemos ha sido obtenido en el contexto de la vida de alguien, como un producto de las esperanzas, los temores y los sueños de alguien. La narrativa es una de las operaciones fundamentales de construcción de sentido que posee la mente; y al parecer es peculiar tanto de los individuos como de la humanidad en su conjunto (Córdoba, 2007, p. 236).

La ficción posee muchísimos elementos que la ponen en valor a la hora de desarrollar estrategias y medios de aprendizaje, siendo en sí misma una forma de aprender sustancial e insustituible, entendida y articulada como una narrativa que estructura pensamientos de forma creativa y que va mucho más allá del concepto de mentira o falsedad. La ficción, cuando se refiere a la construcción simbólica de mundos a través de medios artísticos y creativos, supone una aproximación consciente, meditada, estructurada, y casi siempre articulada de forma compleja, que ofrece una activación del pensamiento y fuerza al creador de ficciones a imaginar nuevos universos y posibilidades, como proponía Eisner (2004) cuando hablaba de la imaginación como posibilitadora de mundos, creadora de nuevos mundos posibles. Sin olvidar que las narrativas de ficción no son otra cosa que una articulación artística de la imaginación.

"La imaginación, esa forma de pensamiento que engendra imágenes de lo posible, también desempeña una función cognitiva de importancia fundamental. La imaginación nos permite probar cosas - de nuevo con el ojo de la mente- sin las consecuencias que podríamos encontrar si tuviéramos que probarlas empíricamente. Ofrece una red de seguridad para experimentar y ensayar (Eisner, 2004, p. 21).” 


\section{METODOLOGÍA}

\section{Procedimiento}

Se ha procedido a desarrollar un análisis reflexivo y teórico de las propuestas que reivindican, desde la disciplina de la educación artística, el uso de narrativas de ficción, desde diversos ámbitos, para construir un relato que demuestre la necesidad y la capacidad de este tipo de prácticas artísticas en la mejora de la docencia a la hora de abordar cuestiones vinculadas con la identidad.

\section{Análisis de datos}

Las prácticas de investigación basadas en las narrativas de ficción han sido utilizadas en las disciplinas vinculadas a las Ciencias Sociales como método de trabajo, para la mejora del desarrollo social y colectivo de grupos de personas, también desde la Investigación Basada en Artes se reivindica de forma más reciente como vía de trabajo muy importante, especialmente en ámbitos educativos.

A través del análisis de algunas propuestas teóricas e investigaciones prácticas desarrolladas y especialmente, mediante la reflexión y profundización en las diferentes metodologías de las narrativas de ficción, como metodología y experiencia pedagógica aplicada en el aprendizaje y la sensibilización, se investiga sobre las posibilidades abiertas que nos ofrecen las acciones educativas basadas en la creación de nuevas narrativas, que partiendo de la ficción, se plantean y replantean las relaciones humanas, el vínculo y la comprensión hacia otras personas, el respeto y la asunción de la diferencia como la normalización de las acciones vitales.

Todo ello tiene como base acciones artísticas educativas y metodologías basadas en las Arts-Based Research o Investigación Basada en Artes, cuyas contribuciones son muy positivas en diversos ámbitos sociales y de la salud, y contribuyen a la mejora del bienestar humano.

\section{RESULTADOS}

Para integrar las prácticas artísticas en las formas de creación de conocimiento "hará falta librarse de algunas dicotomías problemáticas como por ejemplo la separación entre objeto y sujeto, entre realidad y ficción, entre la forma y el contenido" (Lajeunesse, 2007, p. 262). Es decir, la forma en la que el ser humano comprende y entiende el mundo, y especialmente la forma en la que el ser humano delimita y crea su propia identidad. Esta, no es otra cosa que su forma de estar en el mundo y de relacionarse con el resto de los seres humanos. Es un conglomerado de experiencias que pasan por aquellas que entendemos como reales, pero que las que consideramos, ficción, imaginación, son una parte absolutamente conectada e imprescindible para una comprensión global del mundo 
realmente experimentado y vivenciado por una persona. No se puede avanzar en la comprensión y construcción del mundo y de uno mismo, sino se parte de las narrativas de ficción, de la capacidad de imaginarse a uno mismo en otros roles, en otras personas, jugando otros papeles, o proyectándose en el futuro.

Cuando hablamos de identidades, de la propia construcción de la identidad personal, surge de nuevo la duda de la forma en la que vamos definiendo esa identidad y aparece de nuevo la necesidad de construirnos a nosotros mismos como si de un relato de ficción que estamos obligados a reescribir constantemente, se tratara. De nuevo la ficción, entendida bajo esa significación simbólica de creación de narrativas que nos permiten asimilar y comprender el mundo y a nosotros mismos de una forma más significativa, y nos facilitan la creación de conocimiento, estructurándose en sí misma como una experiencia artística sensible de aprendizaje, y por tanto como una experiencia educativa integral y compleja.

"La narración de sí es un intento de reconstruir, siempre a posteriori, una unidad de su propia existencia, no con una objetividad impensable, sino en una búsqueda de sentido $\mathrm{Y}$ de coherencia que no excluye la reinterpretación, sincera incluso, de los acontecimientos. En todo momento, el individuo se siente ligado a su historia, no cesa en su interior de contarse a sí mismo, encontrando los hilos que guían su recorrido en los acontecimientos recientes y, en las situaciones presentes acumulando otros recuerdos que lo llevan a seguir siendo el mismo y a la vez a redefinirse constantemente con el transcurrir de los años. La identidad que el propio individuo se construye y se reconstruye a través de su narración es una ficción sin lugar a dudas, pero se trata del único medio para acercarse a sí mismo, a través de un proceso sin fin que no para de modularse" (Le Breton, 2017, p. 182).

Si en la propia construcción de la biografía existencial, ya elaboramos un complejo de narrativas sobre nosotros mismos, que no se ha materializado en una narrativa estructurada, aunque sí muchas veces en una narrativa vitalmente desarrollada a través de nuestras acciones y de nuestras relaciones, el llevar a cabo una materialización consciente de narrativas, a través de medios artísticos, como puedan ser las narrativas orales, escritas y por supuesto, las narrativas visuales y audiovisuales, supone una toma de conciencia autoreflexiva sobre la propia identidad y establece un proceso de aprendizaje sobre uno mismo, partiendo de la generación de discursos autoetnográficos y autobiográficos.

Mediante el uso de prácticas educativas vinculadas a la creación de narrativas se pueden obtener resultados pedagógicos muy importantes para la construcción de nuevas formas de relación, comprensión e interpretación de aspectos identitarios como son las cuestiones de género, entre muchos otros aspectos que definen a los seres humanos a través especialmente de la vinculación con los demás. No debemos olvidar que la identidad sexual y de género, es ante todo una forma de vinculación a otras personas, una forma de definición de la manera que tenemos de amar, de gozar y de compartir con el otro en 
función de nuestras inclinaciones sexuales. Estas son especialmente conflictivas a nivel social, si no se adecuan a las normas que rigen en una sociedad heteropatriarcal.

Ciertamente a través de la construcción de narrativas visuales y audiovisuales se cubre un ámbito de las relaciones y el conocimiento humano que queda especialmente huérfano desde una orientación más tradicional o positivista, ya que como nos recuerdan algunos investigadores en este ámbito "la ciencia social tradicional tiene dificultades para tratar los elementos sensoriales, emocionales y corporales." (Gorospe, Correa, Jiménez de Aberasturi, y Gutiérrez, 2009, p. 8). De esta forma, mediante el uso de narrativas, narrativas que vinculamos a prácticas y experiencias artísticas y que dotamos de un valor creativo, se desarrollan de forma especialmente apta una serie de valores, capacidades y conocimientos que entroncan con aspectos sensibles del ser humano de una forma mucho más eficaz. Especialmente a la hora de generar procesos de relación empática, hacia la otra persona, facilitando la comprensión de su propia biografía y de su identidad, que a través de la comunicación establecida mediante medios artísticos se hace visible, en ocasiones de forma sutil y en otras de forma descarnada, en función de la necesidad y el carácter del creador.

Esta necesidad de visibilizar la construcción de las identidades propias se lleva a cabo de una manera mucho más eficaz a partir de medios artísticos narrativos, tal y como demuestran algunas investigaciones y aportaciones precedentes y especialmente la obra de artistas que trabajan con estos medios de creación y experimentación. En este sentido medios como la fotografía artística y experimental y el videoarte, se muestran mucho más adecuados para la indagación de cuestiones identitarias, por su alto grado de flexibilidad y la amplitud de dimensiones que poseen como medios creativos en sí mismos. Son miles los casos de artistas que han construido, a través de la fotografía y del vídeo, sin entrar en los que lo han hecho en la dirección, producción y creación de películas cinematográficas, para generar relatos sobre su propia identidad sexual y de género, su afirmación o el cuestionamiento de los propios roles de identidad y género establecidos por la propia demarcación social. Citaremos solamente como ejemplos, casos muy conocidos como los artistas Gilbert y George, que a través de narrativas visuales fotográficas, construyen un universo de autoafirmación identitaria de su homosexualidad mediante recursos artísticos y estéticos, o el caso de Martha Rosler, que a través de la fotografía y el vídeo y creando personajes de ficción encarnados en ella misma, fue capaz de contar tantas verdades y remover pensamientos profundos a través de sus narrativas visuales y audiovisuales.

Un ejemplo perfecto de este tipo de desarrollos de indagación identitaria centrados en el videoarte como forma de exploración identitaria en contextos educativos y como práctica educativa en sí misma, nos lo ofrece el trabajo y la investigación de Arcoba (2016) entre alumnado adolescente. Esta joven investigadora plantea el uso del vídeo, desde una perspectiva artística entre alumnado adolescente, como una narrativa autobiográfica y auto-etnográfica que le permite indagar en la manera en que la creación 
narrativa de ficción video gráfica supone un medio perfectamente adecuado para la exploración identitaria y la asunción de discursos que sería muy difícil articular a través de otros medios, como son aquellos aspectos que tienen que ver con la identidad sexual y de género, con la propia asunción y afirmación del propio género, el cuerpo, la personalidad, la gestión de afectos y sentimientos, etc. Un medio tan proclive a construir discursos artísticos sensibles como la videocreación, articulador de poéticas narrativas identitarias por excelencia, se convierte en un aliado natural de la educación para la consolidación de discursos múltiples y posicionamientos diversos, frente a una visión monocanal del mundo.

A través del análisis de todos estos procesos, prácticas y metodologías didácticas, se comprueba la eficacia y la eficiencia de la Investigación Educativa Basada en las Artes, como forma de generar propuestas de acción pedagógica para contribuir a la mejora y el compromiso social, la lucha por los derechos humanos y la libertad, que contribuye al bienestar general a nivel emocional, psicológico, social y cultural de muchas personas en función de su libre elección de opción sexual y contribuye también a ese bienestar de las personas que se mostraban intolerantes frente a la diversidad de los otros, mejorando su comprensión mediante prácticas empáticas a través de lenguajes artísticos.

\section{CONCLUSIONES}

A partir del análisis de las narrativas de ficción como método pedagógico y centrado en la utilización del arte como medio educativo. Se puede concluir que la educación artística, en todas sus variantes, y como muestra analizada, las narrativas de ficción, contribuyen o pueden contribuir, de forma eficaz y como método creativo, a la asunción de nuevas formas de pensamiento que en la vida "real" y cotidiana muchas personas no se habían planteado, muchas veces por la falta de una interpelación o mediación necesaria.

También es especialmente útil en aquellas personas con una mentalidad más reticente al cambio, la adaptación y los posicionamientos empáticos. Estas prácticas, contribuyen a imaginar mundos posibles y otras posibilidades y posicionamientos que favorecen la posición empática y el cambio de mentalidad y predisponen a una mayor comprensión de opciones y formas de vida diversas, entendiendo finalmente la diversidad como una riqueza vital que nos aporta y nos enriquece colectivamente a todos como seres humanos.

Finalmente, se ha trazado un cuestionamiento teórico sobre el propio concepto de ficción, estableciendo la necesidad de incorporar, cuestionando los valores establecidos entre ficción y no ficción, este tipo de narrativas en las prácticas de aprendizaje y comprensión del mundo y de uno mismo, así como de la relación con los demás. 


\section{Agradecimientos}

Este artículo forma parte del proyecto "Arteari. Arte y diseño para entornos educativos libres de homofobia y transfobia" con referencia UV-INV-AE18-779907 de la Convocatoria de Ayudas para Acciones Especiales de Investigación 2018 del Vicerrectorado de Investigación de la Universitat de València.

\section{REFERENCIAS}

Agra, M.J. (2005). El vuelo de la mariposa: La investigación artístico-narrativa como herramienta de formación. En R. Marín Viadel (Ed.), Investigación en educación artística (pp. 126150). Granada: Universidad de Granada.

Arcoba, M.D. (2016). La videocreación en el área de educación artística. Exploración de la identidad mediante el autorretratoa audiovisual. (Máster Universitario en Profesor/a de Educación Secundaria TFM), Universitat de València, València.

Córdoba, V.Y. (2007). La narrativa visual como metodología del sentido: articulación metodológica e implicaciones terapéutico-educativas/Visual narrative as a methodology of the sense: methodological articulation and therapeutic-educational implications. Arteterapia, 2, 233246.

Eisner, E.W. (2004). El arte y la creación de la mente. Barcelona: Paidós.

Gardner, H. (2014). Estructuras de la mente: la teoría de las múltiples inteligencias (2* ed.). México: Fondo de Cultura Económica.

García Roldán, Á. (2012). Videoarte en contextos educativos las nuevas narrativas audiovisuales y su inclusión curricular en los programas de educación artística desde una perspectiva $a / r / t /$ ográfica. (Phd), Universidad de Granada, Granada.

Goodman, N. (1995). De la mente y otras materias. Madrid: Visor.

Gorospe, J., Correa, J.M., Jiménez de Aberasturi, E., y Gutiérrez, L.P. (2009). El e-portafolio en el proyecto Elkarrikertuz: Las narrativas audiovisuales en el aprendizaje de la cultura escolar y la formación inicial del profesorado reflexivo. Revista de Educación a Distancia, 1-16.

Huerta, R. (2016). Transeducar. Arte, docencia y derechos LGTB. Madrid-Barcelona: Egales.

Huerta, R., y Alonso-Sanz, A. (2015). Educación artística y diversidad sexual. València: Universitat de València.

Le Breton, D. (2017). Desaparecer de sí. Una tentación contemporánea. Madrid: Siruela.

Leavy, P. (2018). Fiction-Based Research. En P. e. Levy (Ed.), Handbook of Arts-Based Research (pp. 190-207). New York: Guilford Press.

Mesías-Lema, J.M. (2008). El murmullo de la circulación de la sangre: la fotografía como medio narrativo en Educación Artística. Arte, Individuo y Sociedad, 20, 69-94.

Samuel-Lajeunesse, J.F. (2007). Nuevas formas literarias para las ciencias sociales: el caso de la autoetnografía. Athenea Digital, 12, 262-271.

Recibido: 28 de marzo de 2019

Recepción Modificaciones: 7 de abril de 2019

Aceptado: 10 de abril de 2019 